\title{
Topology Optimization of Microstructures to Reduce Structural Oscillations
}

\author{
Georgios Stylianos Zymvragakis ${ }^{1}$, Panagiotis Koutsianitis ${ }^{2}$ and Georgios E. Stavroulakis ${ }^{3, *}$ \\ 1 Affiliation 1; s.zymvragakis@gmail.com \\ 2 Affiliation 2; panoskout@gmail.com \\ 3 Affiliation 3; gestavr@dpem.tuc.gr \\ * Correspondence: s.zymvragakis@gmail.com; panoskout@gmail.com; gestavr@dpem.tuc.gr; Tel.: +30 \\ 2821037418 (G.E.S.) \\ Technical University of Crete, School of Production Engineering and Management, Institute of \\ Computational Mechanics and Optimization, GR-73100 Chania, Greece
}

\begin{abstract}
Vibration suppression in a field of frequencies and the creation of band gaps which do not allow the propagation of the waves is studied in this paper by means of microstructures designed through topology optimization. Topology optimization is formulated in frequency domain. Band gap design is based on Floquet-Bloch theory and genetic optimization. The result is appealing in view of modern 3D printing techniques.
\end{abstract}

Keywords: vibration suppression; topology optimization; band gaps; Bloch theory; finite element analysis (List three to ten pertinent keywords specific to the article; yet reasonably common within the subject discipline.)

\section{Introduction}

Mechanical vibrations and oscillations are dynamic phenomena which drastically affect the behavior of a product or a structure. This effect can be stress concentrations that will lead to a reduction in its mechanical properties and possibly even to breakage due to material fatigue as well as to a reduction in the life time of the product.

Various strategies have been studied to reduce oscillations such as the use of anti-vibration structures (sound insulation, dampers, anti-vibration bearings), active control (consists mainly of feedback control systems through a mechanical equipment or a structure eg piezoelectric patches etc.) or structural design which will be able to dampen the propagation of the waves and consequently reduce oscillations.

The aim of all above techniques is to prevent the transmission of oscillation in the structure.

The 'ideal' solution for controlling structures in order to reduce oscillations would be a system with a structure capable of meeting some of the following characteristics:

- High stiffness to be able to respond to static loading

- Ability to reduce oscillations to a fairly wide range of oscillations

- Low density

- High strength

- Ability to work in different operating conditions

- A shape that facilitates construction with either conventional or more modern methods.

All the cases mentioned partially meet some of the above criteria. One method that could cover a very wide range of requirements is the utilization of Band gap areas. Band gaps are areas between a range of frequencies that prevent a wave from propagating at these frequencies. Band gaps are usually attributed to the presence of microstructures or periodic arrangement of inclusions and voids or even the existence of defects. Therefore, a periodic medium with a large band gap can attenuate 
vibrations over a wide range of frequencies. It is important to note that the energy from the oscillation is reflected back to the source through the Bragg scattering mechanism or it is absorbed through a local tuned mass damper mechanism without energy propagation [1].

To create such zones, the Bragg fission method is used, which is produced by changes in the geometry or material of a periodic structure. This method can be used when the grid of periodic microstructures (size of representative unit cells or volumes) is greater than or equal to the wavelength [2].

Homogenization techniques based on Bloch's theory have been developed in 3D periodic microstructures to create three-dimensional lattices that will cut waves at different frequencies [3].

The analysis of Bloch theory has been used within finite element models of two degrees of freedom (beam elements), where the behavior of an entire grid can be accurately assessed by studying only one shell of which it consists. [4] Auxetic microstructures have been studied with twodimensional plane elasticity elements in [5] and enhanced with shunted piezoelectric patches with beam elements in [6]. Usage of classical shape optimization has been tested for the design of band gap structures in [7]

Topology optimization has certain advantages over classical optimal structural design and is able to provide innovative microstructures that can suppress a broader range of waves. The pioneering publication in this field [8] follows a large number of publications, see for example the review article [9] or the two-scale procedure published in [10].

The innovation lies in the synthesis of multiple structures using the theory of band gap to cut off the propagation of the wave in multiple frequency ranges.

The present paper follows recent activity in this area and uses two-material topology optimization for the design of a composite with embedded microstructure leading to band gap formation [11], [12], [13].

The structure of the paper is as follows. The genetic optimization tool is outlined in section two. Modelling of wave propagation and band gaps in mechanical structures and numerical treatment using the finite element method and Bloch theory are presented in the next session. Numerical results are presented in the last part of the paper.

\section{Materials and Methods}

\subsection{Theory of genetic algorithms}

The optimization of the system was carried out using a genetic algorithm (G.A), which is a powerful method of applicable global optimization, able to avoid local minima, which requires only the calculation of the value of the goal function and does not require calculation or even existence of derivatives [14]. The code has been implemented in a programming language in Matlab, to make it easier for the system model, developed in Matlab as well, to work with the genetic algorithm. The basis of each algorithm is the same, the differentiation is in the parameters and constraints of the variables.

\section{Genetic Algorithm Features}

The G.A. rely heavily on imitating the process of evolution as well as the process of heredity in living organisms.

The main functions that take place in organisms are reproduction and mutation.

The basic principle of genetic algorithms is to maintain a population of the problem in the form of coded information and the evolution of the population over time. The evolution of members of the population is based on the laws of natural selection (survival of the fittest) and the recombination of genetic material within the population. The evolving population samples the search space, accumulates information about the areas of good and bad quality solutions and by exchanging pieces of information forms solutions with optimal behavior for the specific problem. 
Genetic algorithms are a method of stochastic optimization that uses many candidate solutions (large population of candidate solutions) at the same time [15]. Thus, the optimum solution is being approached by the best member of the whole population at each iteration.

After creating the initial population, each member of the population is decoded into a candidate solution to the problem and assigned to this solution a "suitability" value through a quality function that gives a measure of the quality of each solution. Then members of the population are selected in pairs to reproduce and form offspring (new solutions). The choice of pairs is made randomly, so that the probability of choosing each solution is proportional to its "suitability". This guarantees that high quality solutions will be selected many times and will be the "parents" for many new solutions, while low quality solutions will contribute less to the new population, with the possibility that they will not be selected for reproduction.

When both parent solutions are selected, their strings are recombined to produce a descending solution using operators that simulate corresponding genetic mechanisms. The main operators used are crossbreeding and mutation. The crossbreeding combines the parent strings to produce an offspring that inherits characteristics of both parents. Crossbreeding, although the main mechanism for finding new solutions, is not able to produce information that does not already exist in the population. The mutation meets this need by introducing new information to the new offspring. The mutation takes place by randomly changing the symbols of the new offspring. Mutation is generally considered to be a secondary but useful operator, which gives a non-zero chance of control and evaluation to any possible solution.

When new solutions are produced, they are considered as the new generation and completely replace the "parents" so that evolution can proceed. Many generations are required until the population converges to the optimal or near solution, with the number of generations increasing according to the difficulty of the optimization problem.

The general form of the steps of a genetic algorithm is given below:

1. (0) Time instant $\mathbf{t}=\mathbf{0}$

2. Solution population initialization $P(t=0)$

3. Population assessment of solutions

4. (1) Time instant $\mathbf{t}+\mathbf{1}$

5. Selection of new population of solutions $P(t+1)$ from the old $P(t)$

6. Application of operators on the new population

7. Assessment of the new population

8. Return to (1) with $\mathbf{t}=\mathbf{t}+\mathbf{1}$

Step 1 is repeated, until the termination criterion is met, with the result that the algorithm gives an optimal solution that satisfies all constraints. The algorithm in the form of pseudocode is as follows:

\begin{tabular}{|c|}
\hline generation $=0$ \\
Initialize \\
Evaluate \\
KeepBest \\
do generation=1, MAXGENS \\
Select \\
Crossover \\
Mutate \\
Report \\
Evaluate \\
\hline
\end{tabular}




\section{Elitist}

enddo

\subsection{Wave propagation using finite elements}

\subsubsection{Bloch - Floquet theory}

Using Bloch's theory (or Floquet for one-dimensional periodic problems) for any structure with iterative periodic microstructures, the change in wavelength along its propagation in the plane does not depend on where the shell is placed inside the grid. For this reason, it is argued that the behavior of an entire grid can be accurately estimated by studying only one shell of which it is composed.

Based on Bloch's theory, this work focuses on the use of square microstructures - shells where the Brillouin band is defined (Figure $4 \mathrm{a}$ ), during which the wave should propagate to the first quarter (triangular purple area) as shown in Figure 4 (b), in order to estimate the propagation of the wave throughout the shell and consequently throughout the grid which consists of the same shell.

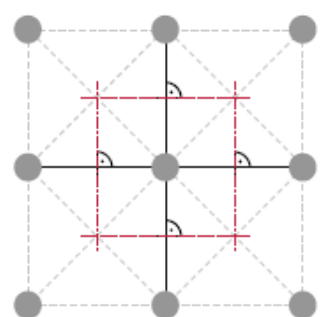

(a)

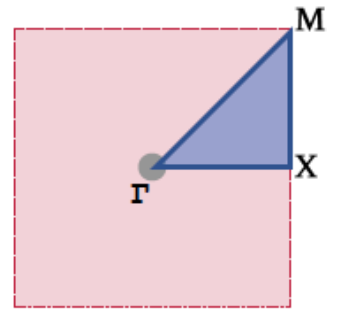

(b)

Figure 4. (a) Construction and (b) illustration of the first Brellouin zone

\subsubsection{Structures and band gap areas}

The vector of the wave $(\mathrm{k})$ contains the information of the total propagation of the wave, within the triangle, and thus the behavior of the waves within the elastic medium, in all directions, can be estimated. Thus, wave propagation can be studied in any shell form, using only the outlined area.

According to the propagation relation, the wave $\mathrm{k}$ is linearly related to the frequency, considering the velocity phase $\left(\mathrm{u}_{\mathrm{p}}\right)$ constant. This hypothesis is satisfactory in one-dimensional wave propagations in a homogenized medium. However, in wave propagation in a two-dimensional medium which may contain various materials, the elements of stiffness and material density do not remain constant. Thus, in this case the velocity phase cannot be considered constant.

In addition, while the elastic properties are constant and the wavelength changes as the frequency changes, the vector of the $\mathrm{k}$ wave differs depending on the shape. By plotting the frequency, as a function of the propagation of the wave within the first Brillouin band, a graph is obtained which shows the range of frequencies at which the wave stops propagating in all three directions. An example of this is shown in Figure 5 where the corresponding area is shown in a gray box. 


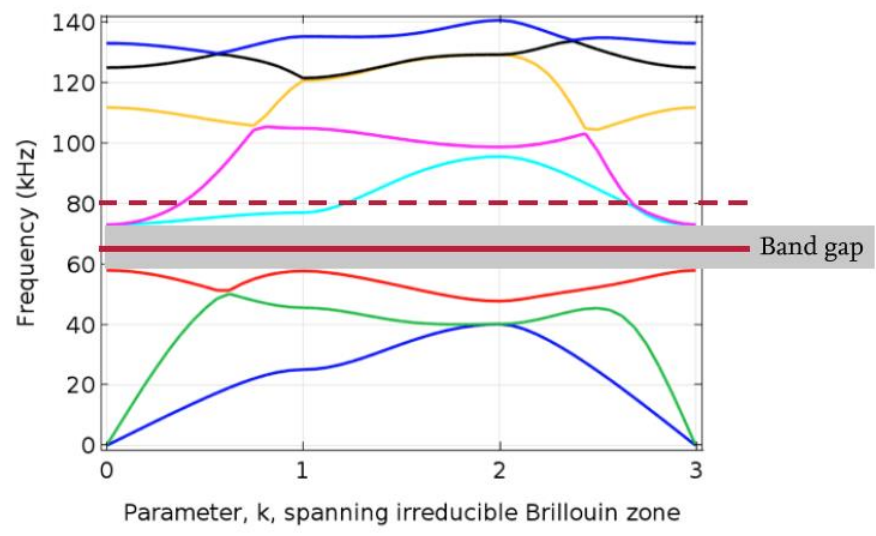

Figure 1. Dispersion curve of first frequencies

\subsubsection{Solving Bloch theory in two-dimensional continua and homogenization}

Periodic microstructures can be thought of as a system of identical parts that are connected to their neighbors on all sides and corners. A classic such lattice is periodic continua of crystal lattice or composite materials. Various examples of mechanical models with periodic conditions can be analyzed from the literature such as waveguides with uniform cross section, conductors, reinforced plates, shells and many other cases.

More specifically, this study focuses on two-dimensional periodic structures, whose properties are homogenized in the 2 dimensions $\mathrm{x}$ and $\mathrm{y}$. This type of structures can be thought of as a grid consisting of rows and columns of rectangular identical sections of length Lx and Ly as shown in Figure 6.

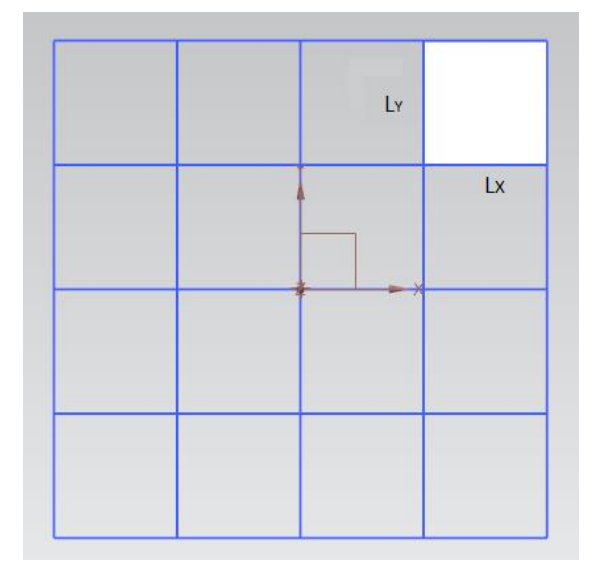

Figure 2. Assembly of many rectangular structures [16]

To study such a structure, the periodicity of a single microstructure was used which is then discretized and studied by the finite element method [17] - [18]. For simple application of these periodic conditions the number of nodes on one side should be equal to the number of nodes on the opposite side. If the periodic lengths Lx and Ly are small enough, the way to distinguish the square segment is by using a single rectangular finite element of four nodes (one on each diagonal) as shown in Figure 7. 


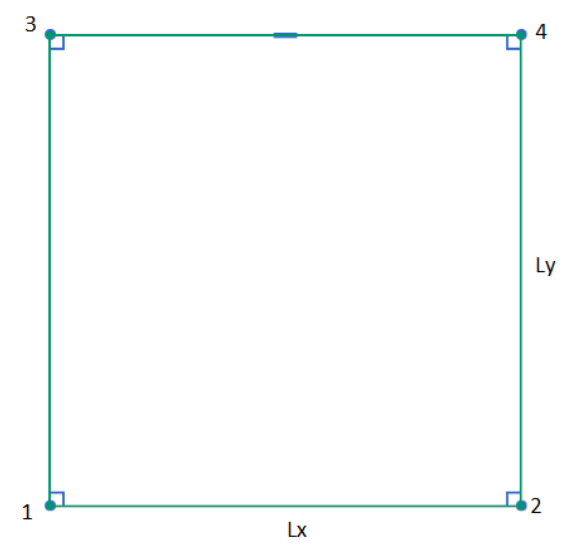

Figure 3. Finite element modeling in a small size segment

Using Figure 7 as a reference, where the microstructure is distinguished by nodes on the diagonal as well as intermediate nodes on the sides of the quadrilateral, we define as degrees of freedom (DOFs) $\mathrm{q}$ of the quadrilateral as the degrees of freedom of movement of its nodes and is defined as

$$
q=\left[\begin{array}{llll}
q_{1}^{T} & q_{2}^{T} & q_{3}^{T} & q_{4}^{T}
\end{array}\right]
$$

where $q_{j}$ is a vector that includes all degrees of freedom of a node of a finite element of the microstructure and in this case $\mathrm{j}$ corresponds to one of the nodes located at the corners of the quadrilateral. Node $\mathrm{j}$ can also be defined as "super-node" which results from the aggregation of all nodes of finite elements that could appear along the thickness. The vector of node loads can be set, respectively, with the displacement vector of the four nodes.

$$
f=\left[f_{1}^{T} f_{2}^{T} f_{3}^{T} f_{4}^{T}\right]
$$

In the case of the free wave propagation study the external loads are not taken into account. The loading vector is nonzero in the case where the charges on the nodes are responsible for propagating the wave from one element to another.

The general equation of wave propagation in the element of Figure 7 can be studied with the following relation:

$$
\left(-\omega^{2} M+i \omega C+K\right) q=f
$$

where the variables $\mathrm{M}, \mathrm{C}$ and $\mathrm{K}$ correspond to the registers of mass, damping and stiffness respectively. The shape of the flat free wave along the structure can be described as the shape of the Bloch [19] Floquet wave. Bloch's theory is well known and widespread mainly in applications of phonon crystal structures and is sometimes referred to as Floquet theory as it represents a generalized constant in the theory of solids for a problem of wave propagation in one direction ( 1 dimensional problem) [20].

Therefore, the free wave propagation can be estimated taking into account the propagation constants

$$
\mu_{\chi}=\kappa_{x} L_{x} \kappa \alpha \iota \mu_{y}=\kappa_{y} L_{y}
$$

which are used to correlate the displacements between the opposite nodes of the periodic elements where based on figure 7 we have:

$$
q_{2}=\lambda_{x} q_{1}, \quad q_{3}=\lambda_{y} q_{1}, \quad q_{4}=\lambda_{x} \lambda_{y} q_{1}
$$

Where

$$
\lambda_{x}=e^{-i \mu_{x}} \quad \lambda_{y}=e^{-i \mu_{y}}
$$

The degrees of freedom of each node can be transformed after the application of the periodic conditions as follows: 


$$
q=\Lambda_{R} q_{1}
$$

Where

$$
\Lambda_{R}=\left[\begin{array}{llll}
I & \lambda_{x} I & \lambda_{y} I & \lambda_{x} \lambda_{y} I
\end{array}\right]
$$

In the absence of external excitation, the equilibrium at node 1 indicates that the sum of the node charges of all elements connected to node 1 is zero. Therefore

$$
\Lambda_{L} f=0
$$

Where

$$
\Lambda_{L}=\left[\begin{array}{lll}
I & \lambda_{x}^{-1} I & \lambda_{y}^{-1} I\left(\lambda_{x} \lambda_{y}\right)^{-1} I
\end{array}\right]
$$

Substituting equation (6) and multiplying both parts by $\Lambda_{\mathrm{L}}$ the equation of free wave propagation will take the form as shown below

$$
\left(-\omega^{2} \bar{M}\left(\mu_{x}, \mu_{y}\right)+i \omega \bar{C}\left(\mu_{x}, \mu_{y}\right)+\bar{K}\left(\mu_{x}, \mu_{y}\right)\right) q=0
$$

Where

$$
\begin{aligned}
\bar{K} & =\Lambda_{L} K \Lambda_{R} \\
\bar{C} & =\Lambda_{L} C \Lambda_{R} \\
\bar{M} & =\Lambda_{L} M \Lambda_{R}
\end{aligned}
$$

The resulting variables are new 'condensed' registers of stiffness, damping and mass respectively, which are of a reduced degree after the calculation of the above operations.

So we have an eigenfrequency problem which can be captured by the following relation

$$
\bar{D}\left(\omega \lambda_{x} \lambda_{y}\right)=\Lambda_{L} M \Lambda_{R}
$$

Where $\mathrm{D}$ is the dynamic stiffness matrix (DSM). In case there are $\mathrm{n}$ degrees of freedom per node, the displacement vectors of each node as well as the forces in each node are of size $n \times 1$, the mass and stiffness registers of each element are $4 n \times 4 n$ while the reduced registers are $n \times n$.

\section{Results}

Geometry is a factor that plays a very important role in creating bands at low frequencies, and can provide solutions that achieve quite satisfactory results. For this reason, the research led to the study of the optimal topology of the structure, based on its geometric characteristics.

The aim of this section is to model an optimization problem using classical optimization algorithms (specifically with a genetic algorithm) in order to design microstructures to create wave propagation cut-off zones in specific frequency bands. This method allows the two different materials to be placed in such a way as to lead to the creation of band gaps in specific frequency bands.

The specific problems to be solved are problems of two materials. This makes it easier for us to use a genetic algorithm, as the problem to be solved is not the placement or not of material by changing its density but the placement of one or the other material. A square two-dimensional microstructure was used to model the problem.

This problem is also a problem of two materials, the properties of which are defined by Table 1 . In addition, the theory of two-dimensional square finite elements, flat stress (plane stress elements) was used.

The optimization problem that has been solved and will be commented on below is: Creating a band gap in a specific frequency range. 


\subsection{Creating a band gap in a specific frequency range}

Of particular interest is the study for the creation of band gaps in specific frequency bands [21] where the propagations of waves under these frequencies are cut off, which we define and we do not know to which eigenfrequencies they can correspond. For this reason an optimization code was created which enables the algorithm to calculate what these frequencies are each time they are in the range we are interested in, e.g. $j$ and $j+1$ and to try to increase the gap between them.

If in the frequency range there were more than 1 interconnected frequencies, then the frequency $\mathrm{j}$ was calculated which was closer to the lower limit set and the design variables were modified in order to increase the distance of this frequency from the next one $(j+1)$. To avoid unsatisfactory solutions, a penalty operator was used, which "expelled" the algorithm from the optimal solution if the range was not greater than or equal to the required.

The cases studied were 3 and are the following:

- Achieve band gap between $5 \mathrm{kHz}-6.5 \mathrm{kHz}$

- Achieve band gap between $8 \mathrm{kHz}-9 \mathrm{kHz}$

- Achieve band gap between $9.5 \mathrm{kHz}-11.5 \mathrm{kHz}$

In all cases we have a maximization problem with $F(\vec{x})=g_{i+1}(\vec{x})-g_{i}(\vec{x})$ where $g_{i}(\vec{x})$ is the eigenfrequency near the lower limit we have set.

The optimization problem is described as below:

$$
\max _{\vec{x}} F(\vec{x}) \text { with } \vec{x}=\left[x_{1}, x_{2}, \ldots, x_{400}\right] \quad \text { (Objective optimization function) }
$$

Under restrictions:

- For band gap between $5 \mathrm{kHz}-6.5 \mathrm{kHz}$

$$
\begin{array}{ll}
g_{i}(\vec{x}) \leq 5 \mathrm{kHz} \text { with } i=1,2, \ldots, n \quad \text { (Anisotropic constraints) } \\
g_{i+1}(\vec{x}) \geq 6.5 \mathrm{kHz} \text { with } i=1,2, \ldots, n & \text { (Anisotropic constraints) }
\end{array}
$$

- For band gap between $8 \mathrm{kHz}-9 \mathrm{kHz}$

$$
\begin{array}{ll}
g_{i}(\vec{x}) \leq 8 \mathrm{kHz} \text { with } i=1,2, \ldots, n \quad & \text { (Anisotropic constraints) } \\
g_{i+1}(\vec{x}) \geq 9 \mathrm{kHz} \text { with } i=1,2, \ldots, n & \text { (Anisotropic constraints) }
\end{array}
$$

- For band gap between $9.5 \mathrm{kHz}-11.5 \mathrm{kHz}$

$$
\begin{array}{cc}
g_{i}(\vec{x}) \leq 9.5 \mathrm{kHz} \text { with } i=1,2, \ldots, n \quad \text { (Anisotropic constraints) } \\
g_{i+1}(\vec{x}) \geq 11.5 \mathrm{kHz} \text { with } i=1,2, \ldots, n \quad \text { (Anisotropic constraints) }
\end{array}
$$

For all cases:

$$
\vec{x}_{j}=0 \quad \text { or } \quad \vec{x}_{j}=1 \quad j=1,2,3, \ldots, 400 \quad \text { (Equality Constraints) }
$$

The population size is set to 50 members, that is, 50 different possible solutions.

The maximum number of generations, which denote the number of total iterations, is set to 50 . 
In the first case the goal was to achieve a construction capable of damping the oscillations in the frequency range $5 \mathrm{kHz}-6.5 \mathrm{kHz}$, ie a band of $1500 \mathrm{~Hz}$. Using the genetic algorithm, and with the methodology previously described, the results obtained are shown in Figure 9. Here for each finite element the design variable $x$ takes two values, $x=0$ for the soft material or $x=1$ for the hard material.
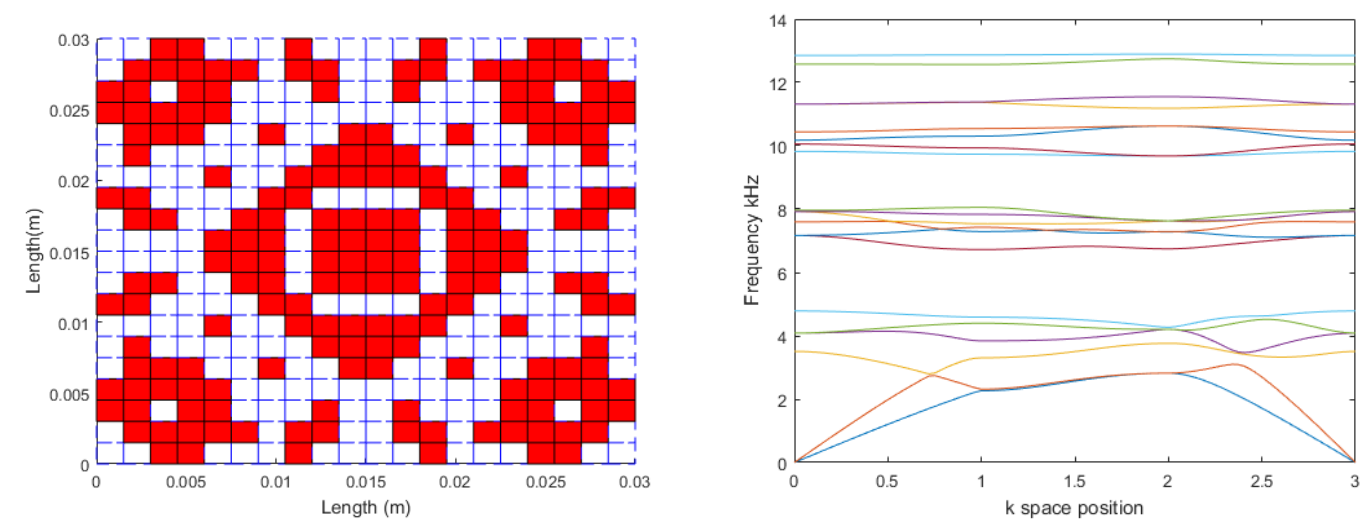

Figure 4. (a) Optimal construction and (b) dispersion curve for optimization between $5-6.5 \mathrm{kHz}$

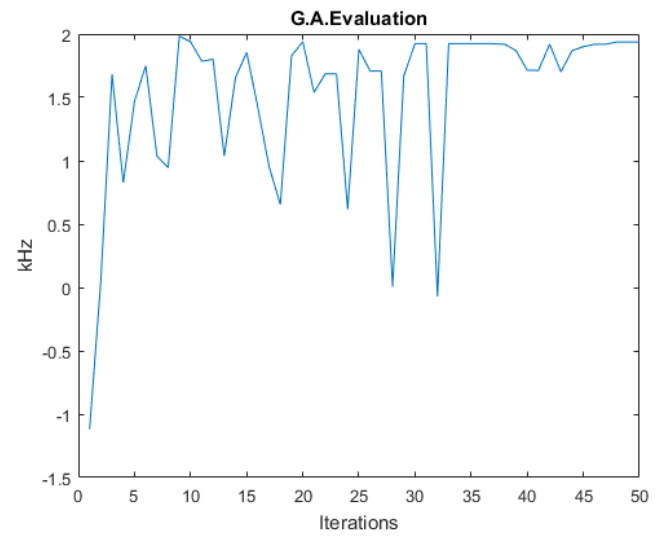

Figure 5. Convergence diagram for optimization between $5-6.5 \mathrm{kHz}$

The algorithm that was developed, achieves with great accuracy and success the creation of band gaps in the defined frequency range. The distribution of soft (white) - hard (red) material amounts to $50 \%$.

The next investigation was between $8 \mathrm{kHz}-9 \mathrm{kHz}$. The results of this optimization are shown in Figure 11. As shown in Figure 11, the material distribution is $>75 \%$ steel. The band gap that was intended to be achieved, to a large extent was created, with the band exceeding the $1 \mathrm{kHz}$ that was set as the upper limit and reaching close to $1.9 \mathrm{kHz}$. 

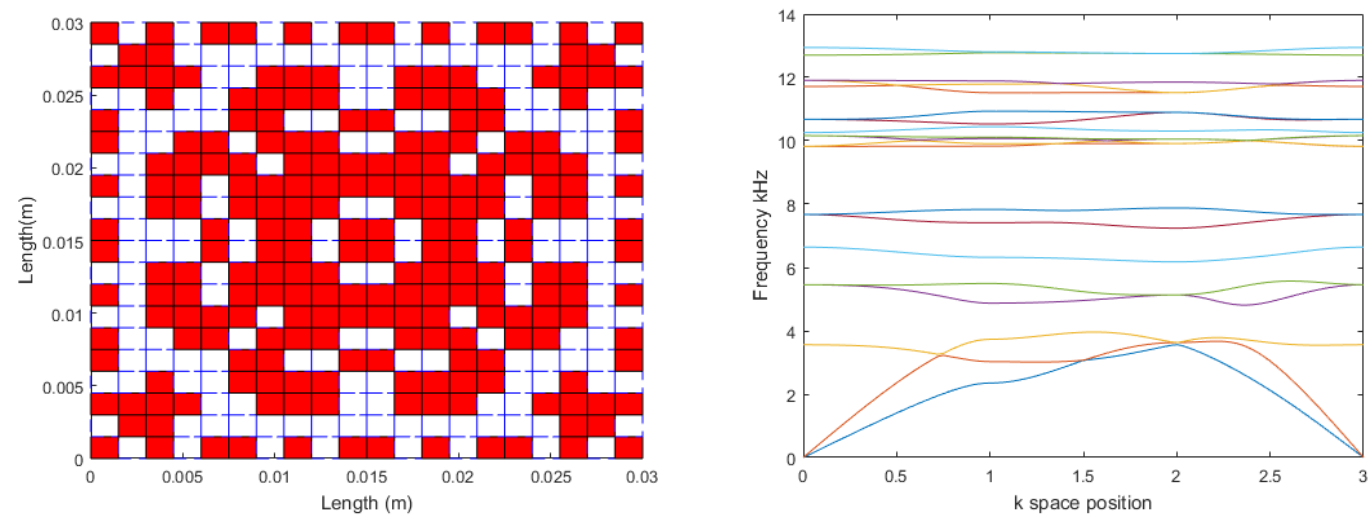

Figure 6. (a) Optimal construction and (b) dispersion curve for optimization between $8-9 \mathrm{kHz}$

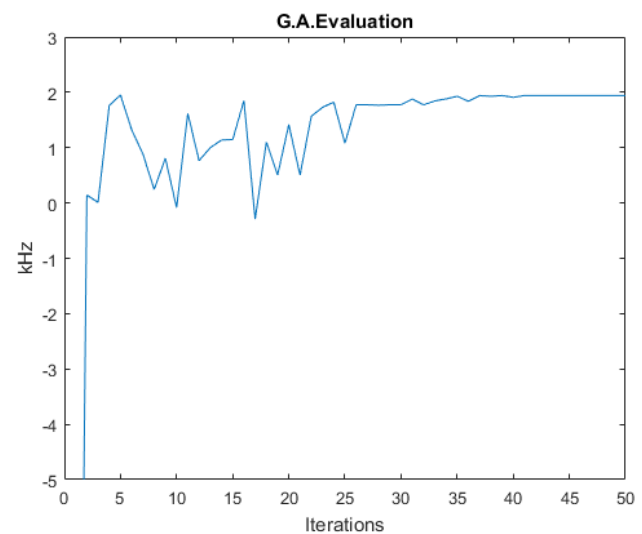

Figure 7. Convergence diagram for optimization between $8-9 \mathrm{kHz}$

Finally, the band gap that was decided to be investigated was between $9.5 \mathrm{kHz}-11.5 \mathrm{kHz}$. A key reason was that while the previous two cases were done to select two random bands, it was observed that in both cases after about $9.5 \mathrm{kHz}$ no band gap appeared nearby. This is how the modeling was done, and the following results were created.

Based on Figure 13, a large band gap is created in the frequency range of interest, as well as a distribution of $60 \%$ steel (red) and $40 \%$ rubber (white). This created a frequency gap in areas that the previous two cases could not achieve.
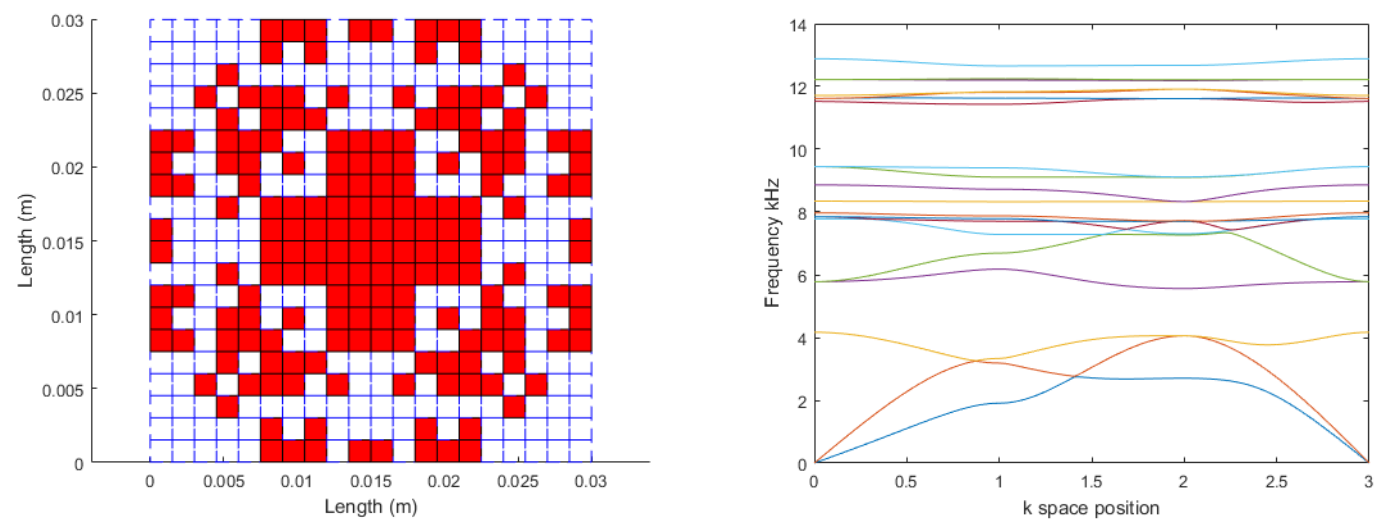

Figure 8. Optimal construction and dispersion curve for optimization between $9.5-11.5 \mathrm{kHz}$ 


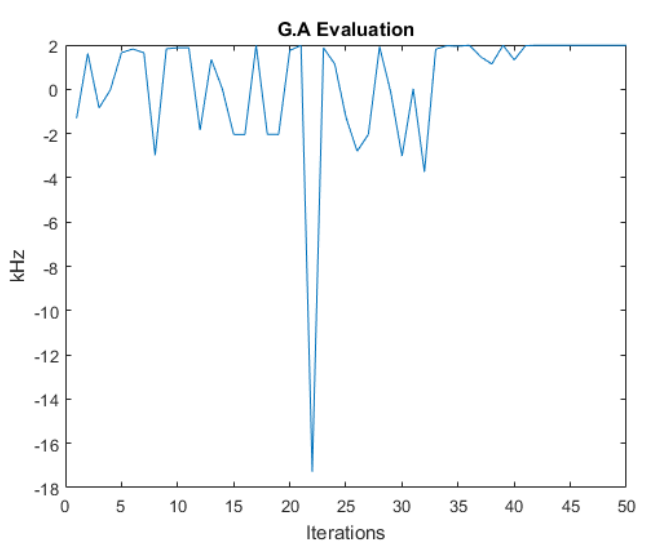

Figure 9. Convergence diagram for optimization between $9.5-11.5 \mathrm{kHz}$

The specific methodology for optimizing the topology of the construction enabled us to implement structures that work in the desired frequency range and dampen the oscillations and not only be limited to increasing the range between two known eigenfrequencies. In addition, with the modern method of production of constructions and products, it becomes possible to create such constructions (two-material 3D printers).

On the other hand, this method is not able to work with a single material, and this is because it fails to create a continuum in the constructions (it creates many islands) and it is practically impossible to make any natural microstructure. The use of hybrid topological optimization techniques could be used [22].

\subsection{Creation of larger structures, composition of different microstructures}

Finally, in this subsection the behavior of structures using the above optimal microstructures which cover the space in the form of grids will be studied and the effect of these grids on the wave propagation under harmonious loading will be calculated.

First, the grid is presented, consisting of the microstructure that was optimized in order to have a band gap between $5 \mathrm{kHz}-6.5 \mathrm{kHz}$. The grid which consists of $5 \times 5$ same unit cell, is shown in Figure 15 and its size is $0.15 \times 0.15 \mathrm{~m}$. The charge applied is on the entire left side, with a charging width of $100 \mathrm{~N}$, application of boundary anchorage conditions on the upper and lower sides and the point at which the displacement will occur where the frequency diagram will occur is located right in the center of the square of the grid.

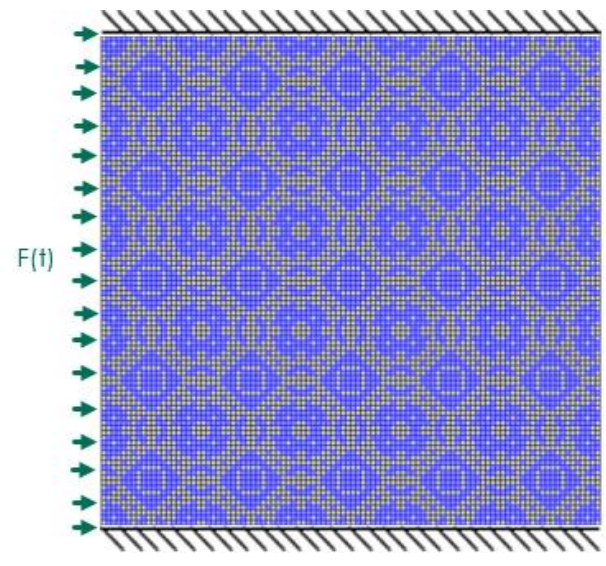

Figure 10. Grid display size $0.15 \times 0.15 \mathrm{~m}$ for the case $5-6.5 \mathrm{kHz}$

The following are the grids and frequency responses of the other two microstructures (8-9.5 and 9.5-11.5 kHz respectively). The data of charges, limit values and measuring points are exactly the 
same in the next two cases. In addition, it is important to mention that in all 3 cases the point studied is approximately at the same point making sure it is on an area where there is hard material (steel).
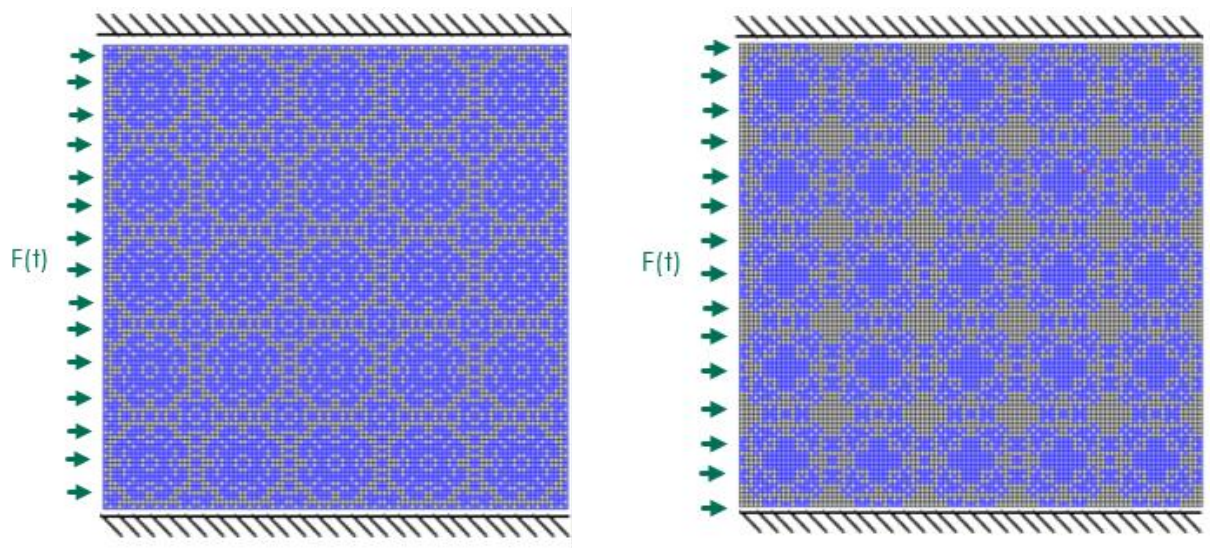

Figure 11. Constructions for band gap between $8-9 \mathrm{kHz}$ and $9.5-11.5 \mathrm{kHz}$ respectively

Figure 17 shows the graph of the response of the three different structures in the frequency domain. And in these cases some 'bellies' are created in the areas where the band gaps appeared, based on the microstructures used. However, in some cases the results do not seem to have the accuracy of the constructions with the microstructures presented in a previous section. The reason is probably that the accuracy of a $5 \times 5$ construction is not enough. However, the efficiency of the methods for studying the behavior of an entire grid from a single microstructure is considered quite satisfactory.

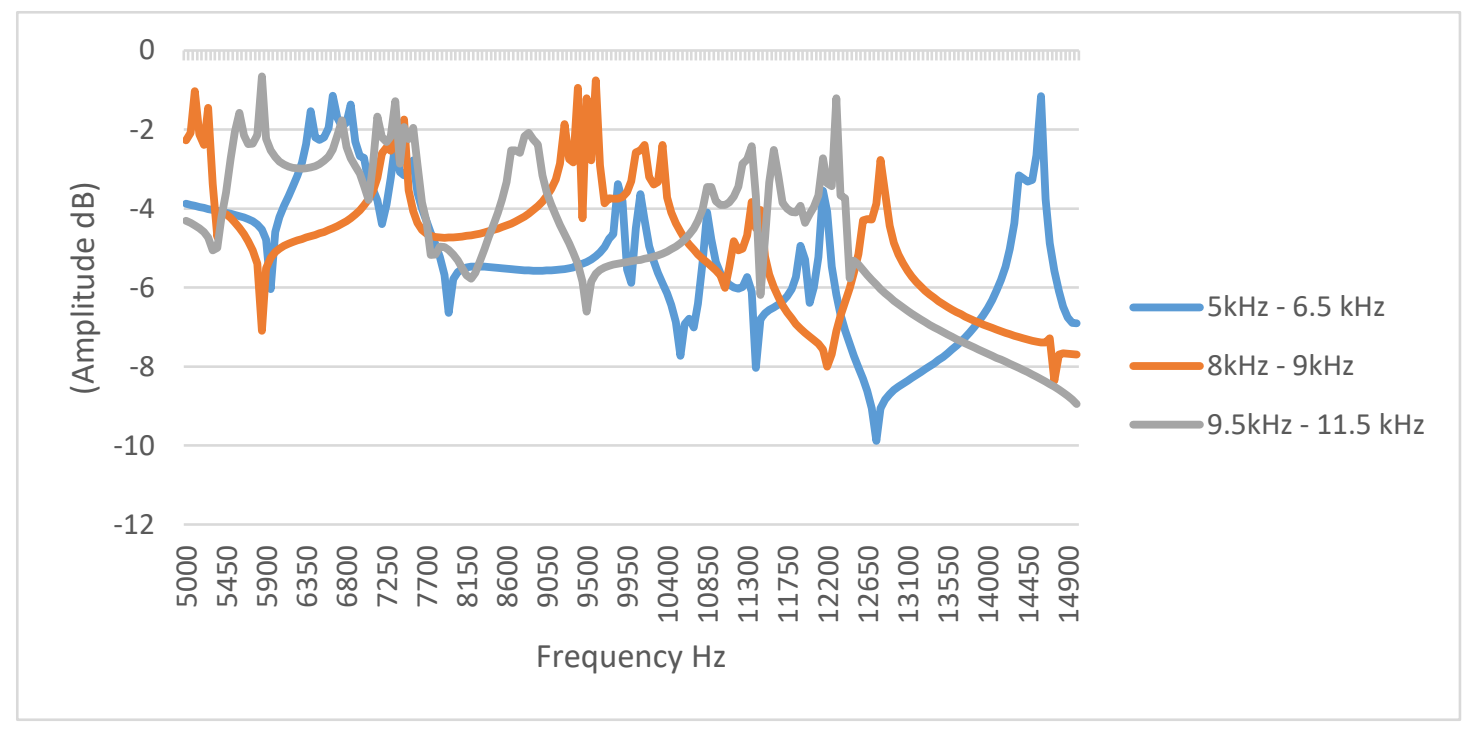

Figure 12. Response in the field of frequencies of the three investigations

The interesting question that arises from the investigations is how a structure that consists of 3 consecutive grids of the same microstructure would behave, and how the one with 3 grids of different structures. More specifically, how the dynamic behavior of the construction changes and whether a combination of "cutting" of multiple eigenfrequencies can be done depending on the type of microstructure at each point and whether this works as a multiple, combination filter. For this reason, the above problem was modeled where the three microstructures were initially placed in series, as shown in Figure 18 and in the process only the third microstructure was used to compare the results. It is worth mentioning that in all cases the exact same measuring point was used, which is located near the right end of the construction, the loads and boundary conditions are the same as in the 
previous cases, as well as it was chosen to model only the third microstructure as this is which will be entered last and the point had to belong to the same unit cell.

The composite structure constructed is shown in Figure 18. In the same way a structure was modeled using microstructure 3 where three identical structures were placed in series.

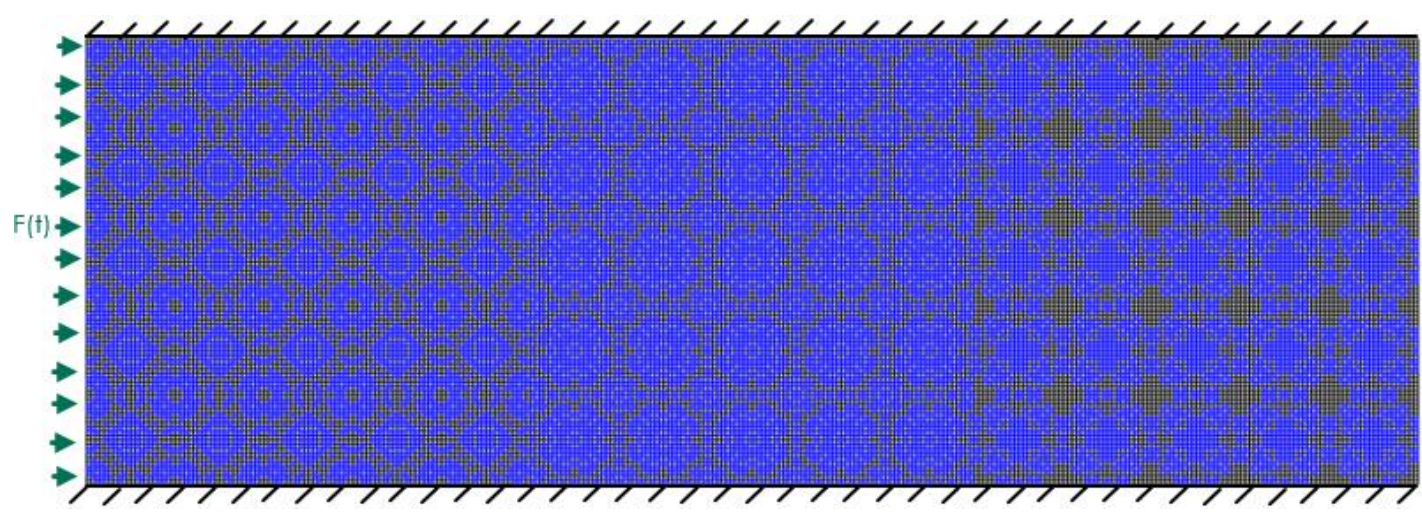

Figure 13. Structure composition with 3 different microstructures

Figure 19 shows the response of the complex construction as well as the construction with the three same structures. From the graph of the composite construction we observe that in comparison with the simple construction in the frequencies of interest it keeps the amplitudes of the displacements very low and essentially cuts the propagation of the wave. On the contrary, in the simple construction that consists only of the third microstructure, although it follows the graph of the simple 3rd grid with different oscillation widths, these remain at quite high levels compared to the complex.

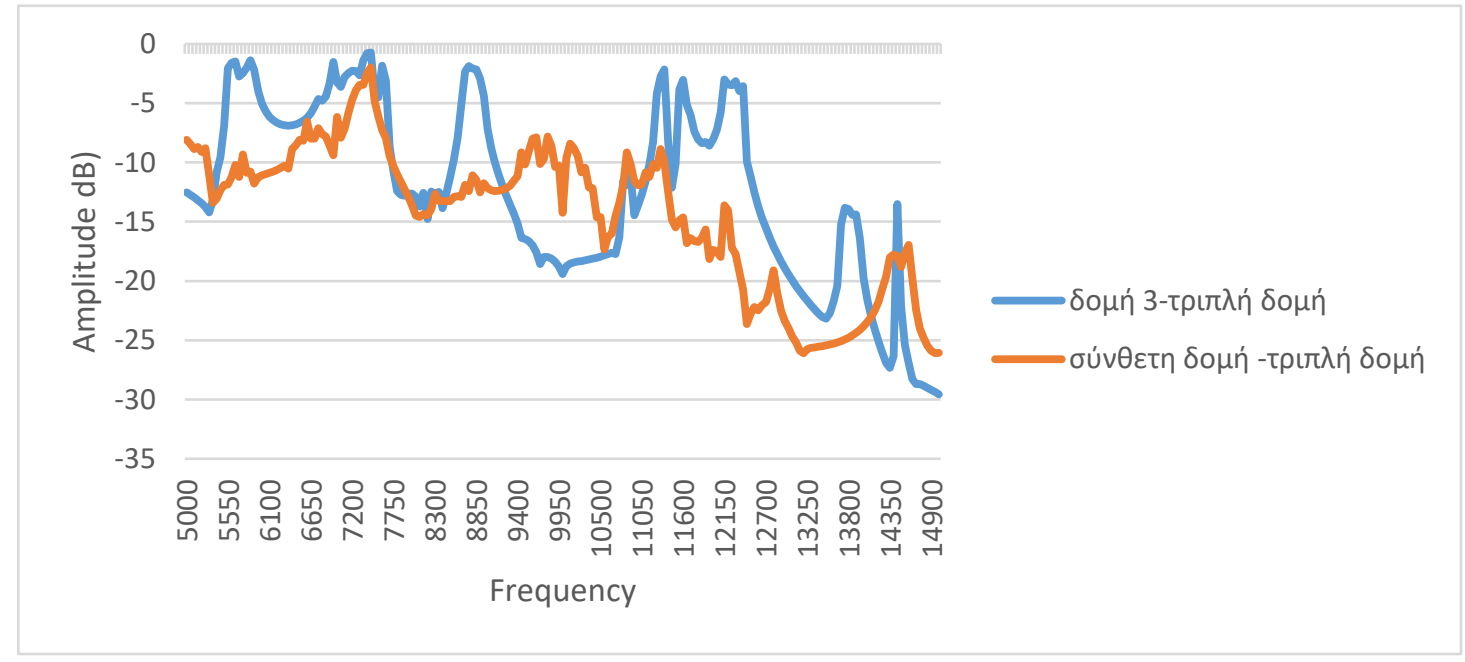

Figure 14. Comparison of simple structure response with complex

The conclusion is that with the use of Bloch theory, and classical optimization algorithms, microstructures can be created that are able to reduce the oscillations in a range of frequencies, and with their further composition to be a filter that can works in combination on the frequencies that interest us.

\section{Discussion}

A basic and essential approach to the response of a structure to dynamic loads is its analysis in the field of frequencies. This produces information about a range of frequencies, as well as 
approximates the physical characteristics (eigenfrequencies- eigenmodes) of the structure. In order to design for the oscillation reduction, an algorithm was developed, which based on Bloch theory and using periodic conditions allows information about the frequency bands that prevent the propagation of the wave. As presented in the results, based on this theory, microstructures were created such as to cut off the propagation of the wave over a wide frequency range.

Based on this method, the necessary microstructures had to be created, which would then be part of a structure and modify the range of frequencies of interest.

The change of geometry is the one that has the greatest influence on the creation of the areas in which the wave will not be able to propagate. For this reason, using classical optimization algorithms, the density and material distribution were modified in order to create the optimal construction design. The algorithm used was a nature-based optimization algorithm (genetic algorithm). As a result, geometries were created that absorb oscillations in desired frequency ranges.

Author Contributions: “Conceptualization, P.K. and G.E.S.; software, GS.Z. and P.K.; investigation, GS.Z.; writing - original draft preparation, GS.Z.; writing - review and editing, GS.Z. and P.K.; supervision, G.E.S.; All authors have read and agreed to the published version of the manuscript."

\section{Appendix A}

Table 1. Elastic properties of materials

\begin{tabular}{|c|c|c|}
\hline $\begin{array}{c}\text { Material } \\
\text { properties }\end{array}$ & Rubber & Steel \\
\hline Density $\left(\mathrm{kg} / \mathrm{m}^{3}\right)$ & 1000 & 7800 \\
\hline $\begin{array}{c}\text { Young's } \\
\text { modulus (Pa) }\end{array}$ & $5 \times 107$ & $200 \times 109$ \\
\hline Poisson's ratio & 0.4 & 0.25 \\
\hline
\end{tabular}

\section{References}

1. Brillouin, L. Wave Propagation In Periodic Structures; McGraw-Hill Book Company, 1946.

2. Mace, B. R.; Duhamel, D.; Brennan, M. J.; Hinke, L. Finite element prediction of wave motion in structural waveguides. Journal of the Acoustical Society of America 2005, 117, p.p. 2835-2843.

3. Patil, G. U.; Matlack, K.H. Effective property evaluation and analysis of three-dimensional periodic lattices and composites through Bloch-wave homogenization. Journal of the Acoustical Society of America 2019, 145, Issue 3.

4. Wu, Z. J.; Wang, Y. Z.; Li, F.M. Analysis on band gap properties of periodic structures of bar system using the spectral element method. Waves in random and complex media 2013, 23, p.p. 349-372.

5. Koutsianitis, P.I.; Tairidis, G.K.; Drosopoulos, G.A. Conventional and star-shaped auxetic materials for the creation of band gaps. Archive of Applied Mechanics 2019, 89, p.p. 2545-2562. https://doi.org/10.1007/s00419-019-01594-1

6. Koutsianitis, P.I.; Tairidis, G.K.; Stavroulakis, G.E. Shunted piezoelectric patches on auxetic microstructures for the enhancement of band gaps. Archive of Applied Mechanics 2020. https://doi.org/10.1007/s00419-020-01804-1

7. Wormser, M.; Wein, F.; Stingl, M.; Körner, C. Design and Additive Manufacturing of 3D Phononic Band Gap Structures Based on Gradient Based Optimization. Materials 2017, 10 , 1125. doi:10.3390/ma10101125

8. Sigmund, O.; Jensen, J.S. Systematic design of phononic band gap materials and structures by topology optimization. Philosophical Transactions of the Royal Society of London 2003. Series A: Mathematical, Physical and Engineering Sciences, 361(1806), p.p. 1001-1019. https://doi.org/10.1098/rsta.2003.1177

9. Yi, G.; Youn, B.D. A comprehensive survey on topology optimization of phononic crystals. Struct Multidisc Optim 2016, 54, p.p. 1315-1344. 
10. Liang, X.; Du, J. Design of phononic-like structures and band gap tuning by concurrent two-scale topology optimization. Structural and Multidisciplinary Optimization 2020, 61, p.p. 943-962.

11. Han, X.K.; Zhang, Z. Bandgap design of three-phase phononic crystal by topological optimization. Wave Motion 2020, 93, 102496. https://doi.org/10.1016/j.wavemoti.2019.102496

12. Xua, W.; Ninga, J.; Lina, Z.; Qia, W.; Liua, H.; Wang, W. Multi-objective topology optimization of twodimensional multi-phase microstructure phononic crystals. Materials Today Communications 2020, 22, 100801. https://doi.org/10.1016/j.mtcomm.2019.100801

13. Liu, Z.F.; Wub, B.; He, C.F. Systematic topology optimization of solid-solid phononic crystals for multiple separate band-gaps with different polarizations. Ultrasonics 2016, 65, p.p. 249-257.

14. Mace, B.R.; Manconi, E. Modelling wave propagation in two-dimensional structures using finite element analysis, Journal of Sound and Vibration 2008, 318, p.p. 884-902.

15. Michalewicz, Z. Genetic Algorithms + Data Structures = Evolution Programs, 3rd ed; Springer: Verlag Berlin Heidelberg, New York, 1996.

16. Floquet, G. Sur les equations differentielles lineaires a coefficients periodiques. Annales scientifiques de l'E.N.S. 1883, 12, 47-88.

17. Bedford, A; Drumheller, D.S. Introduction to elastic wave propagation, 1rd ed.; John Wiley \& Sons Ltd: Chichester, United Kingdom, 1996.

18. Nazarchuk, Z.; Skalskyi, V.; Serhiyenko, O. Acoustic Emission; Springer International Publishing: Cham, Switzerland, 2017.

19. Theocaris, P.S.; Stavroulakis, G.E.; Panagiotopoulos, P.D. Negative Poisson's ratios in composites with starshaped inclusions: a numerical homogenization approach. Archive of Applied Mechanics 1997, 67, p.p 274286.

20. Kaminakis, N.T.; Drosopoulos, G.A.; Stavroulakis, G. E. Design and verification of auxetic microstructures using topology optimization and homogenization. Archive of Applied Mechanics 2015, 85, p.p. 1289-1306.

21. Koutsianitis, P. Design of microstructure of smart materials and structures using optimization. Doctoral dissertation, Technical University of Crete, Department of Production Engineering and Management, Greece, 04/02/2020.

22. Goldberg, D.E. Genetic Algorithms in Search, Optimization, and Machine Learning; Addison-Wesley: Reading, Massachussets, USA, 1989. 